Original article

\title{
EICOSAPENTAENOIC ACID PROVOKES STRONGER IN VITRO ANTIADIPOGENIC EFFECT THAN DOCOSAHEXAENOIC ACID IN DIFFERENTIATED 3T3-L1 CELLS
}

\author{
E. VACHKOVA ${ }^{1}$, P. YONKOVA ${ }^{2}$, N. GRIGOROVA ${ }^{1}$, ZH. IVANOVA $^{1}$, \\ G. PENCHEV ${ }^{2}$, B. BIVOLARSKI ${ }^{1}$, S. STANILOVA ${ }^{3}$ \& I. P. GEORGIEV ${ }^{1}$ \\ ${ }^{1}$ Department of Pharmacology, Animal Physiology and Physiological Chemistry, \\ Faculty of Veterinary Medicine, Stara Zagora, Bulgaria; ${ }^{2}$ Department of Veterinary \\ Anatomy, Histology and Embryology, Faculty of Veterinary Medicine, Stara Zagora, \\ Bulgaria; ${ }^{3}$ Department of Molecular Biology, Immunology and Genetics, Faculty of \\ Medicine, Trakia University, Stara Zagora, Bulgaria
}

\begin{abstract}
Summary
Vachkova, E., P. Yonkova, N. Grigorova, Zh. Ivanova, G. Penchev, B. Bivolarski, S. Stanilova \& I. P. Georgiev, 2018. Eicosapentaenoic acid provokes stronger in vitro antiadipogenic effect than docosahexaenoic acid in differentiated 3T3-L1 cells. Bulg. J. Vet. Med, 21, No 4, 397-405.

The comparative studies of eicosapentaenoic acid (EPA) and docosahexaenoic acid (DHA) effects on the amount of lipid droplets (LD) and within adipocytes are limited. In this study, 3T3-L1 mouse embryo fibroblasts (ATCC ${ }^{\circledR}$ CL-173 ${ }^{\mathrm{TM}}$ ) were expanded up to fifth passage. At the stage of growth arrest, the cells were treated with EPA and DHA separately and in combination at $100 \mu \mathrm{g} / \mathrm{mL}$ for 2 days. Oil Red O staining protocol, subsequent extraction with isopropanol and spectrophotometric determination of absorbed dye were used to establish the amount of intracellular lipid droplets deposition. While DHA administration had no significant effect on reduction of LD intracellular deposition, the EPA treatment decreased optical density (OD) significantly $(\mathrm{P}<0.05)$. Furthermore, a synergic effect of combined application of both PUFAs was not observed. In conclusion, EPA provoked stronger antiadipogenic effect than DHA suggesting that EPA administration would be more effective in already existing obesity.
\end{abstract}

Key words: 3T3L1, antiadipogenic effects, EPA, DHA

\section{INTRODUCTION}

Over the past decades, obesity and related chronic diseases such as type 2 diabetes, dyslipidaemia, atherosclerosis and hypertension are growing worldwide. The common pathogenetic mechanism of these abnormalities is a transient condition known as metabolic syndrome. The quality of dietary fat and the content of longchain omega-3 polyunsaturated fatty acids (LC-PUFAs) - docosahexaenoic acid 
(DHA; C22:6n-3) and eicosapentaenoic acid (EPA; C20:5n-3) in particular are of major importance. Some authors established that they affected plasma levels of triglycerides and high-density lipoprotein cholesterol, which explains to a great extent their protective role on cardiovascular disease, and at the same time stimulate the body anti-inflammatory defense mechanisms (Ruxton et al., 2004).

Recently, a number of studies were conducted, showing the main role of adipose tissue in the development of metabolic syndrome (Laclaustra et al., 2007; Slawik \& Vidal-Puig, 2007; Dodson et al., 2011). Thus in turn allows reducing the negative effects of obesity by modulating its secretory function by $n-3$ LCPUFAs.

The 3T3-L1 mouse adipocytes are widely used as a system model for study of adipocyte differentiation (Rosen et al., 1979) and exploration of metabolic disorders of adipose tissue for more than 30 years. Peroxisome proliferator-activated receptors (PPARs) are transcription factors that are bound and activated by fatty acids and fatty acid derivatives. It is assumed that the expression of PPAR $\gamma$ regulated genes depends on the length and extent of fatty acids unsaturation which is also relevant to the lipid droplets formation during adipocytes differentiation (Bernlohr et al., 1997; Takahashi \& Ide, 1999). It is acknowledged that EPA is an agonist and a regulator of PPAR $\gamma$ gene expression, and that prolonged treatment with EPA appears to inhibit its expression in certain fat depots (Chambrier et al., 2002).

Some authors reported that EPA-rich feeding reduced the amount of adipose tissue and inhibited the development of obesity in rats (Rustan et al., 1993). With regard to adipocytes themselves, EPA induces the expression of genes related to the biosynthetic processes in mitochondria and oxidative metabolism, increasing lipids catabolism (Flachs et al., 2005; Flachs et al., 2009). In addition, EPA is also relevant to the synthesis of some adipokines such as leptin and adiponectin (Flachs et al., 2009). They improve insulin sensitivity (Kamohara et al., 1997; Yamauchi et al., 2001), although the data on their plasma concentrations and deposition in adipocytes are contradictory. Most recent studies have revealed that in cell cultures of human adipocytes, EPA and DHA increased the secretion of adiponectin by $88 \%$ and $47 \%$ respectively, 48 hours after the treatment (Tishinsky et al., 2011).

Despite the numerous studies in last few years, investigations on the differential effect of EPA and DHA on the amount of the adipocytes and the formation of the lipid droplets within, are limited. Therefore further investigations on differentiated 3T3-L1 cell cultures are needed to clarify the effect of the independent and co-administration of n-3 LC-PUFAs on adipocytes and their usage in the prevention and treatment of metabolic syndrome.

\section{MATERIALS AND METHODS}

3T3-L1 mouse embryo fibroblasts (ATCC ${ }^{\circledR}$ CL-173 ${ }^{\mathrm{TM}}$ ) were expanded up to fifth passage in basic culture media (basal media-BM), consisting of Dulbecco's Modified Eagle Medium (DMEM - low glucose), $10 \%(\mathrm{v} / \mathrm{v})$ foetal bovine serum (FBS), L-glutamine and antibiotic solution (penicillin G, streptomycin, amphotericin B) (Sigma), at $37{ }^{\circ} \mathrm{C}$, in a humidified $5 \%$ $\mathrm{CO}_{2}$ atmosphere, till obtaining 95\% confluence, in $25 \mathrm{~cm}^{2}$ tissue flasks. Then the cells were reseeded in 24-well plates at a density of $5 \times 10^{4} / \mathrm{mL}$, cultured at same conditions for three days and left another 
24 hours to achieve density-dependent inhibition of cell division.

At this stage, depending on the treatment, two major experimental groups were formed, each of them comprising several subsets ( 5 wells each, $\mathrm{n}=5$ ):

1) Group of non-induced cells

- BM (basal medium), in which the cells were grown only in basal medium for 21 days;

- $\quad \mathrm{CM}$ (control medium) - the cells were grown in culture medium consisting of DMEM (high glucose), 10\% FBS, L-glutamine and antibiotic solution (penicillin G, streptomycin, amphotericin B). This group was used to differentiate the degree of spontaneous induction;

- AMM (adipocyte maintainance medium) consisting of DMEM (high glucose), 10\% FBS, L-glutamine, 10 $\mu \mathrm{g} / \mathrm{mL}$ insulin and antibiotic solution (penicillin G, streptomycin, amphotericin B), in which the cells were grown for 21 days;

\section{2) Group of induced cells}

Cells from all subsets of this main group were treated for three days with adipocyte inducing medium (AIM) containing DMEM (high glucose), 10\% FBS, L-glutamine, $0.1 \mathrm{mM}$ 3-isobutyl-1-methylxanthine (IBMX), $0.05 \mathrm{mM}$ indomethacin, $1 \mu \mathrm{M}$ dexamethasone, $10 \mu \mathrm{g} / \mathrm{mL}$ insulin and antibiotic solution (penicillin G, streptomycin, amphotericin B). After this induction period, the cells were divided into respective subgroups and treated as follows:

- CM: treated with control medium alone (CM) for 18 days;

- AMM: treated with maintenance medium alone (AMM) for 18 days. After this period, the cells were cultured in control medium (CM) for 2 additional days;

- EPA: treated with maintenance medium for 18 days, and cultured in control medium (CM) supplemented with EPA at concentration of $100 \mu \mathrm{g} / \mathrm{mL}$ for 2 additional days;

- DHA: treated with maintenance medium for 18 days, and cultured in control medium (CM) supplemented with DHA at a concentration of $100 \mu \mathrm{g} / \mathrm{mL}$ for 2 additional days;

- EPA+DHA: treated with maintenance medium for 18 days, and cultured in control medium (CM) with the addition of EPA and DHA at a concentration of $100 \mu \mathrm{g} / \mathrm{mL}$ for 2 additional days.

After finishing the cycle of adipogenesis ( 3 days in induction media +18 days in maintenance media) and treatment of cells with PUFAs either separately or in combination, the Oil Red O staining protocol (Sigma), subsequent extraction with isopropanol and spectrophotometric determination of absorbed dye was applied to establish the amount of intracellular lipid droplets deposition.

At the end of the trial, cells from experimental and control groups were washed twice with PBS and fixed with $10 \%$ (vol/vol) neutral buffered formalin for $30 \mathrm{~min}$ at room temperature. During fixation a fresh working solution of Oil Red O (Sigma) was prepared by mixing three parts $0.5 \%$ Oil Red O in isopropanol stock solution with two parts of dd $\mathrm{H}_{2} \mathrm{O}$, allowed to stay at room temperature for 10 min and filtered through filter paper to remove the unresolved stain particles (Yang et al., 2011). Fixed cells were washed twice with PBS, and each well was filled up with $300 \mu \mathrm{L}$ Oil Red O working solution and incubated for $30 \mathrm{~min}$ at room temperature. The stain was dis- 
Eicosapentaenoic acid provokes stronger in vitro antiadipogenic effect than docosahexaenoic acid in...

carded, the cells were triple washed with distilled water, and images were taken using an inverted Telaval Carl Ziess JENA (Germany) microscope equipped with a MDCE-5 high resolution ocular camera (China). Further, immediately after washing procedure, the wells were dried and each stained well filled up with $1 \mathrm{~mL} 100 \%$ isopropanol for $10 \mathrm{~min}$ at room temperature to extract the accumulated Oil Red O stain from the intracellular lipid droplets. The optical densities (OD) of collected eluates were measured at $490 \mathrm{~nm}$ using Evolution ${ }^{\mathrm{TM}} 300 \mathrm{UV}$ Vis spectrophotometer (Thermo Scientific, USA) and the grade of absorbance was presented as mean value of tree consecutive measurements for each sample.

\section{Statistical analysis}

Preliminary results were processed by statistical software Statistica v. 6.1 (StatSoft Inc., 2002). Descriptive statistics methods were used to determine the means and standard deviations ( \pm SD). Post hoc LSD test was used, to evaluate the antiadipogenic features of PUFAs on the mean values of the observed index in differentiated 3T3-L1 cell cultures at three levels of statistical significance: $\mathrm{P}<0.05 ; \mathrm{P}<0.01$ and $\mathrm{P}<0.001$.

\section{RESULTS}

During the experiment the cells were successfully differentiated into mature (adult) adipocytes (Fig. 1 and 2).

Spontaneous adipogenesis in uninduced cells group (Fig. 1, BM and CM) was observed only in single cells. In CM, many cells deposited small, oval, located around the nucleus lipid droplets (LDlipid droplets).

Over $80 \%$ of the cells in AMM were in adipogenesis. Part of the cells was observed to increase the volume of the LD and tended to aggregate. In addition, mean \pm SD OD values in this subgroup $1.154 \pm 0.17$ were significantly $(\mathrm{P}<0.001)$ higher compared to $\mathrm{BM}(0.560 \pm 0.02)$ and CM (0.702 \pm 0.04$)$ (Fig. 3).

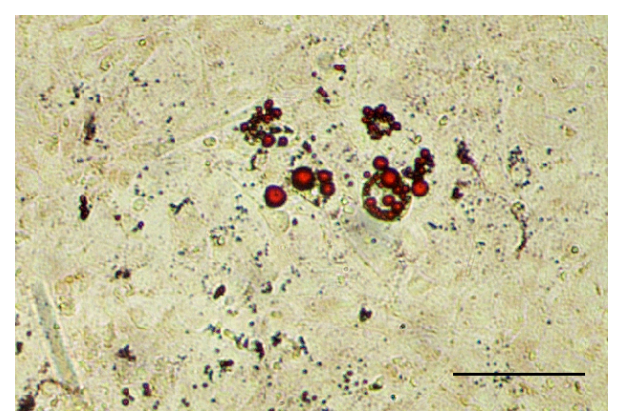

Basal medium (BM)

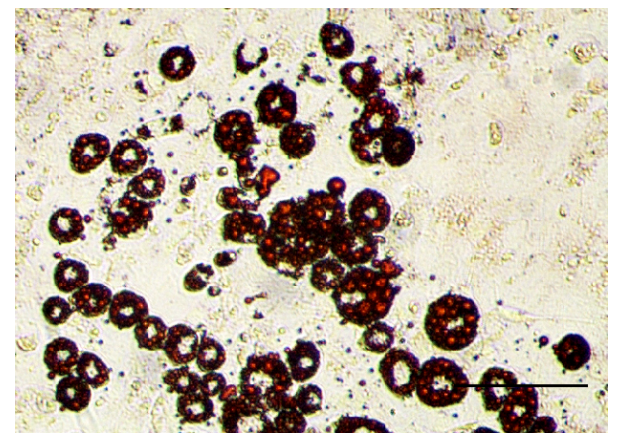

Control medium (CM)

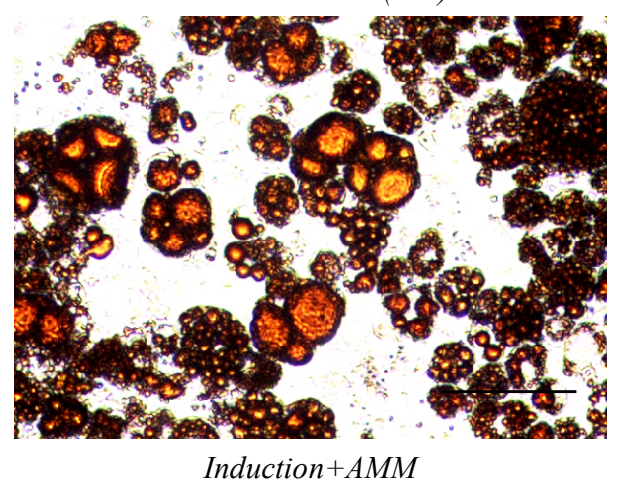

Fig. 1. Microscopic images of 3T3-L1 from uninduced (BM and $\mathrm{CM}$ ) and induced untreated (Induction + AMM) cell groups at the end of the experimental period, Oil Red O staining. Scale bar $200 \mu \mathrm{m}$. 


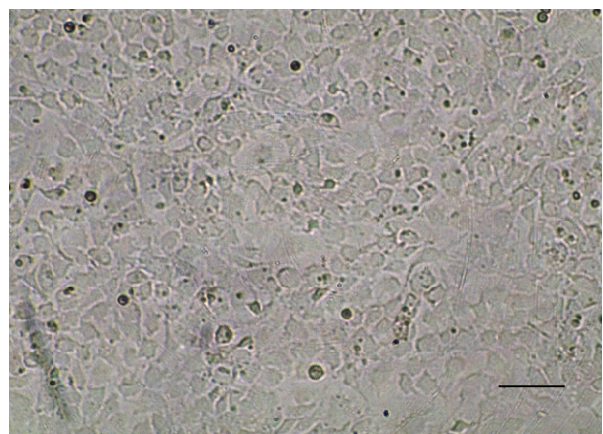

Before induction

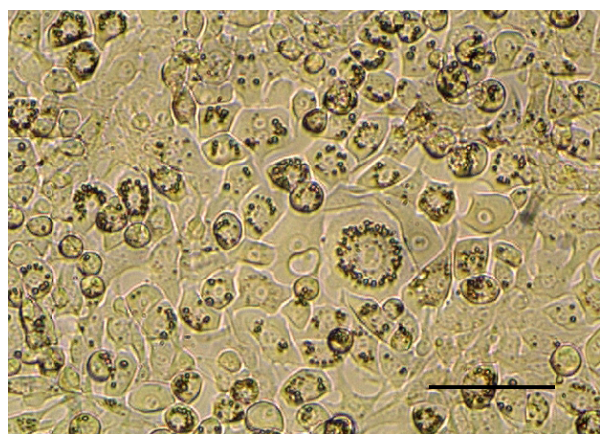

Six days after induction

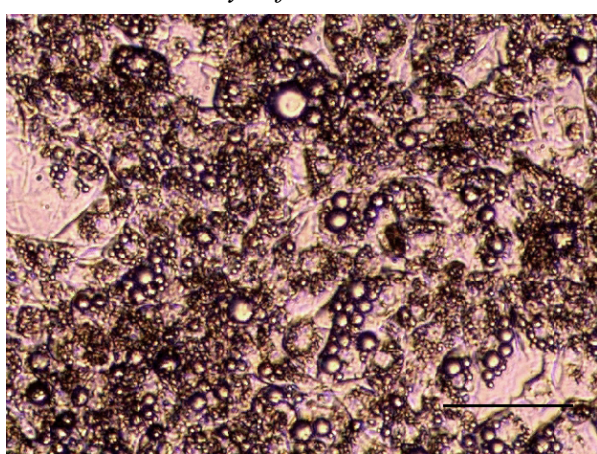

At the end of induction

Fig. 2. Light-microscopic pictures of 3T3-L1 of induced cells, native preparations. Scale bar $200 \mu \mathrm{m}$.

In the group of induced untreated cells, OD values (Fig. 3) were higher for AMM (1.348 \pm 0.15$)$ compared to $\mathrm{CM}$ $(1.176 \pm 0.08)(\mathrm{P}<0.05)$; this was also the highest mean $\mathrm{OD}$ value among all other groups.
In the induced and treated cells group, a decrease in OD after EPA application alone $(1.130 \pm 0.05 ; \mathrm{P}<0.01)$ or in combination with $\mathrm{DHA}(1.154 \pm 0.13 ; \mathrm{P}<0.01)$ was established, compared to the induced and non-treated cells (AMM). Although the DHA treatment had no significant effect on reduction of LD intracellular deposition, the average OD values $(1.271 \pm 0.07)$ in this subgroup were lower compared to AMM. Furthermore, the EPA administration alone decreased significantly $\mathrm{OD}(\mathrm{P}<0.05)$ as compared to the group treatedonly with DHA.

\section{DISCUSSION}

The major finding of this study is that EPA provokes stronger in vitro antiadipogenic effect than DHA in differentiated 3T3-L1 cells. The combined effect was similar to that of EPA suggesting no synergism of both omega-3 PUFAs concerning their antiadipogenic properties.

Non-induced and induced, untreated cells groups were used to monitor the adipogenesis process. Gradual significant increase of OD values in non-induced cells (BM, CM and AMM) was a quantitative expression of the adipogenesis depending on the presence of high levels of glucose and insulin in the medium. Although some authors suggest that insulin is the most powerful regulator of adipogenesis (Klemm et al., 2001), the reported maximum significantly higher OD values in untreated and induced by AMM cells, demonstrated the presence of additional factors affecting the degree of cells induction.

According to the traditional protocols, induction of differentiation in the stage of cell confluence by hormonal cocktail consisting of insulin, DXM, and 3-isobutyl-1methylxanthine, preadipocytes can diffe- 


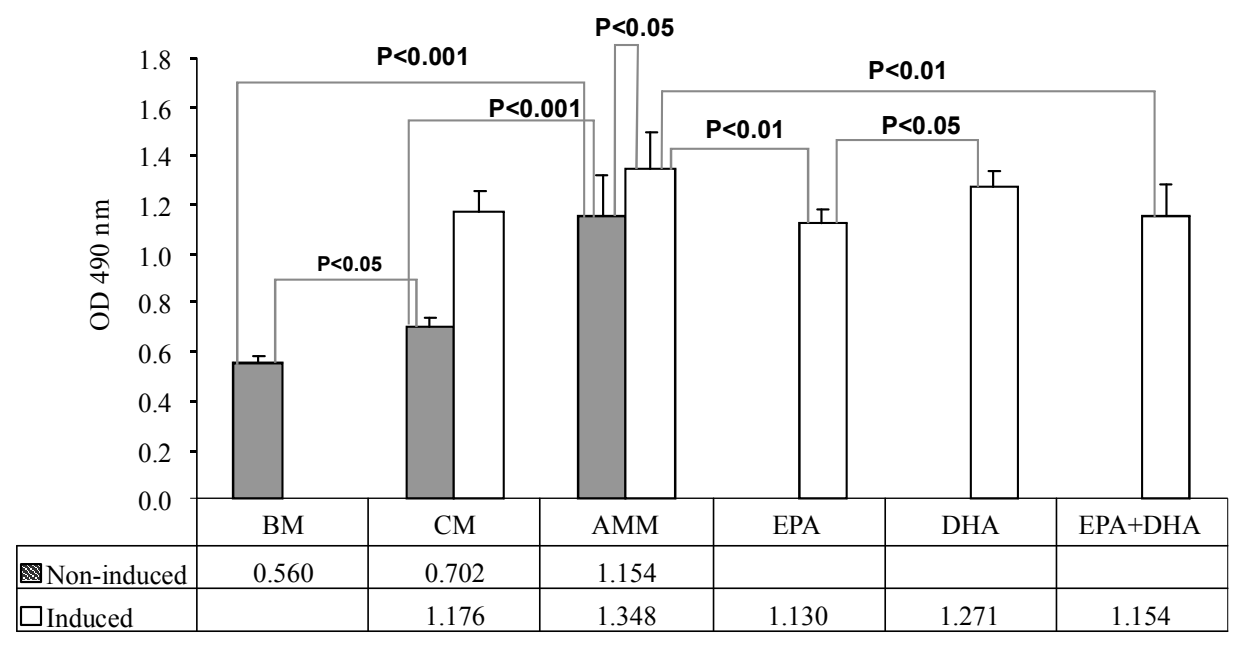

Fig. 3. Optical density at $490 \mathrm{~nm}$ (mean $\pm \mathrm{SD} ; \mathrm{n}=5$ for all groups)

rentiate into mature adipocyte cells, expressing specific adipocyte genes and accumulating triacylglycerol lipid droplets (Cornelius et al., 1994). The synergistic interaction in combined activation of these three signal pathways in the initial phase of differentiation is essential for the full development process of adipogenesis (Gregoire et al., 1998; Ntambi \& YoungCheul, 2000; Petersen et al., 2008), and its ultimate goal is the activation of $\operatorname{PPAR} \gamma$. In this aspect, our results in the untreated groups proved the successful differentiation of preadipocytes into adipocytes and confirmed the data of the above mentioned authors.

It is known that n-3 PUFAs directly affect the activity of PPARs (Flachs et al., 2009). Although EPA is an agonist and positive transcriptional gene regulator of the PPAR $\gamma 1$ (Chambrier et al., 2002), prolonged treatment with EPA in differentiating adipocytes decreased significantly the intracellular deposition of lipid droplets in our experiments. In this respect, during the process of cell differentiation into adipocytes, a mechanism protecting cells from intracellular lipid deposition is probably activated. This defense mechanism is presumably similar to the effect of EPA on some genes involved in the expression of lipid catabolism (Flachs et al., 2005; 2009). The results of the present study showing significant decrease in OD after EPA treatment alone compared to untreated and induced cells (AMM), indirectly supports this assumption. It is interesting to note that OD values in this subgroup were very similar and not statistically different compared to induced CM and non-induced AMM. Probably EPA affected not only the expression of lipolytic enzymes, but also the insulin pathway for maintaining adipogenesis in its later stages, preventing further deposition of lipid droplets in cells. On the other hand, if there was a superimposition of these two mechanisms maybe the rate of decrease would be more strongly expressed. Therefore, additional studies are needed to further elucidate this hypothesis.

There are still disagreements regarding the mechanism of DHA action. Some authors found that DHA added at the time of 
3T3-L1 differentiation, inhibited this process by inducing cells apoptosis and thus reduced their volume and weight respectively (Kim et al., 2006; Prostek et al., 2014). On the other hand, it was noted that PUFA, including DHA, suppressed the intracellular deposition of triglycerides in 3T3-L1 during the same period (Madsen et al., 2005). The reported trend of OD decrease in this subgroup compared to induced and non-treated AMM showed that DHA exhibited an antiadipogenic effect even if applied after the period of cell differentiation. Furthermore, in addition to the DHA influence on the cell apoptosis, Kim et al. (2006) noted an enhancement of the lipolytic processes in already mature adipocytes as a result of treatment of the cells with this polyunsaturated fatty acid. In this respect, our results rather confirmed that DHA reduced the intracellular deposition of lipid droplets even after the period of cell differentiation.

Based on obtained results it could be concluded that EPA had a stronger antiadipogenic effect compared to DHA. According to some authors, EPA and DHA affect preadipocyte differentiation in a different way. For example, DHA treatment of 3T3-L1 during the differentiation was not associated with activation of the inflammatory response and the authors suggested that this DHA effect even improves lipid deposition in adipose tissue (Ganesan et al., 2012). On the other hand, EPA modulates lipid metabolism by induction of the lipolytic genes expression and suppresses those associated with adipogenesis in 3T3-L1 (Lee et al., 2008).

In the present study the combined cell treatment with both PUFAs significantly decreased fat droplets deposition in the cells, but obtained values were similar to those after EPA treatment alone, and that was mainly due to the effect of EPA. Finally, the comparative analysis on the adipogenic effect of both PUFAs showed significant antiadipogenic effect of EPA, either alone or co-applied with DHA. Synergism between EPA and DHA was not observed and probably the established changes after combined application were due to the EPA activation influence on the lipolytic factors. This study raised new questions about the mechanism of action of EPA and DHA. Therefore, additional studies are needed to further elucidate the gene expression levels of some key enzymes and factors involved in lipogenesis and lipolysis to clarify the molecular mechanisms of the stronger antiadipogenic effect of EPA.

The present results confirmed the antiadipogenic properties of omega-3 PUFAs on differentiated 3T3-L1 cell culture. However, EPA provokes stronger antiadipogenic effect than DHA suggesting that EPA administration would be more effective in already existing obesity.

\section{ACKNOWLEDGEMENTS}

Sincere thanks to Prof. Diana Ivanova and Assoc. Prof. Yoana Kiselova, Department of Biochemistry, Molecular Medicine and Nutrigenomics, Faculty of Pharmacy, Medical University, Varna, for the kindly provided cell line 3T3-L1 (ATCC ${ }^{\circledR}$ CL-173TM); Research Project 17/12 of FVM and Research Project 2013 of Central Scientific and Research Laboratory, Trakia University, Boyana Rashkova - student.

\section{REFERENCES}

Bernlohr, D. A., N. R. Coe, M. A. Simpson \& A.V. Hertzel., 1997. Regulation of gene expression in adipose cells by polyunsaturated fatty acids. Advances in Experimental Medicine and Biology, 422, 145-156. 
Eicosapentaenoic acid provokes stronger in vitro antiadipogenic effect than docosahexaenoic acid in...

Chambrier, C, J. P. Bastard, J. Rieusset, E. Chevillotte, D. Bonnefont-Rousselot, P. Therond, B. Hainque, J. P. Riou, M. Laville \& H. Vidal, 2002. Eicosapentaenoic acid induces mRNA expression of peroxisome proliferator-activated receptor gamma. Obesity Research, 10, 518-525.

Cornelius, P., O. A. MacDougald \& M. D. Lane, 1994. Regulation of adipocyte development. Annual Review of Nutrition, 14, 99-129.

Dodson, M. V., P. S. Mir, G. J. Hausman, L. L. Guan, M. Du, Z. Jiang, M. E. Fernyhough \& W. G. Bergen, 2011. Obesity, metabolic syndrome, and adipocytes. Journal of Lipids, doi:10.1155/2011/721686.

Flachs, P., O. Horakova, P. Brauner, M. Rossmeisl, P. Pecina, N. Franssen-van Hal, J. Ruzickova, J. Sponarova, Z. Drahota \& C. Vlcek, 2005. Polyunsaturated fatty acids of marine origin upregulate mitochondrial biogenesis and induce betaoxidation in white fat. Diabetologia, $\mathbf{4 8}$, 2365-2375.

Flachs, P., M. Rossmeisl, M. Bryhn \& J. Kopecky, 2009. Cellular and molecular effects of n-3 polyunsaturated fatty acids on adipose tissue biology and metabolism. Clinical Science (London), 116, 1-16.

Ganesan, M., M. Clevenger, C. Desouza \& V. Saraswathi, 2012. Effects of omega-3 fatty acids on 3T3-L1 preadipocyte differentiation and inflammatory response. Abstracts from the Arteriosclerosis, Thrombosis and Vascular Biology Scientific Sessions, 32, Abstract 142.

Gregoire, F. M., C. M. Smas \& H. S. Sul, 1998. Understanding adipocyte differentiation. Physiological Rewiews, 78, 783-809.

Kamohara, S., R. Burcelin, J. L. Halaas, J. M. Friedman \& M. J. Charron, 1997. Acute stimulation of glucose metabolism in mice by leptin treatment. Nature, 389, 374-377.

Klemm, D. J., J. L. Wayne, P. Watson, A. Nesterova, J. B. Reusch, M. L. Goalstone \& B. Draznin, 2001. Insulin-induced adipocyte differentiation: Activation of CREB rescues adipogenesis from the arrest cau- sed by inhibition of prenylation. The Journal of Biological Chemistry, 276, 28430 28435.

Kim, H. K., M. A. Della-Fera, J. Lin \& C. A. Baile, 2006. Docosahexaenoic acid inhibits adipocyte differentiation and induces apoptosis in 3T3-L1 preadipocytes. Journal of Nutrition, 136, 2965-2969.

Laclaustra, M., D. Corella \& J. M. Ordovas, 2007. Metabolic syndrome pathophysiology: The role of adipose tissue. Nutrition, Metabolism and Cardiovascular Diseases, 17, 125-139.

Lee, M. S., I. S. Kwun \& Y. Kim, 2008. Eicosapentaenoic acid increases lipolysis through up-regulation of the lipolytic gene expression and down-regulation of the adipogenic gene expression in 3T3-L1 adipocytes. Genes \& Nutrition, 2, 327-330.

Madsen, L., R. K. Peterson \& K. Kristiansen, 2005. Regulation of adipocyte differentiation and function by polyunsaturated fatty acids. Biochimica et Biophysica Acta (BBA) - Molecular Basis of Disease, 1740, 266286.

Ntambi, J. M. \& K. Young-Cheul, 2000. Adipocyte differentiation and gene expression. Journal of Nutrition, 130, 3122-3126.

Petersen, R. K., L. Madsen, L. M. Pedersen, P. Hallenborg, H. Hagland, K. Viste, S. O. Døskeland \& K. Kristiansen, 2008. Cyclic AMP (cAMP)-mediated stimulation of adipocyte differentiation requires the synergistic action of Epac- and cAMPdependent protein kinase-dependent processes. Molecular and Cellular Biology, 28, 3804-3816.

Prostek, A., M. Gajewska, D. Kamola \& B. Bałasińska, 2014. The influence of EPA and DHA on markers of inflammation in 3T3-L1 cells at different stages of cellular maturation. Lipids in Health and Disease, 13, doi:10.1186/1476-511X-13-3.

Rosen, O. M., C. J. Smith, A. Hirsch, E. Lai \& C. S. Rubin, 1979. Recent studies of the 3T3-L1 adipocyte-like cell line. Recent Progress in Hormone Research, 35, 477 499. 
Rustan, A. C., B. E. Hustvedt \& C. A. Drevon, 1993. Dietary supplementation of very long-chain n-3 fatty acids decreases whole body lipid utilization in the rat. Journal of Lipid Research, 34, 1299-1309.

Ruxton, C. H., S. C. Reed, M. J. Simpson \& K. J. Millington, 2004. The health benefits of omega- 3 polyunsaturated fatty acids: a review of the evidence. Journal of Human Nutrition and Dietetics, 17, 449-459.

Slawik, M. \& A. J. Vidal-Puig, 2007. Adipose tissue expandability and the metabolic syndrome. Genes \& Nutrition, 2, 41-45.

Takahashi, Y. \& T. Ide, 1999. Effect of dietary fats differing in degree of unsaturation on gene expression in rat adipose tissue. $A n-$ nals of Nutrition and Metabolism, 43, 8697.

Tishinsky, J. M., D. W. Ma \& L. E. Robinson, 2011. Eicosapentaenoic acid and rosiglitazone increase adiponectin in an additive and PPAR $\gamma$-dependent manner in human adipocytes. Obesity, 19, 262-268.

Yamauchi, T., J. Kamon, H. Waki, Y. Terauchi, N. Kubota \& K. Hara, 2001. The fatderived hormone adiponectin reverses insulin resistance associated with both lipoatrophy and obesity. Nature Medicine, 7, 941-946.
Yang, M. T., J. Fu, Y. Wang, R. A. Desai \& C. S. Chen, 2011. Assaying stem cell mechanobiology on microfabricated elastomeric substrates with geometrically modulated rigidity. Nature Protocols, 6, 187-213.

Paper received 29.11.2016; accepted for publication 06.02.2017

\section{Correspondence:}

Ekaterina Vachkova, $\mathrm{PhD}$

Animal Physiology Unit,

Department of Pharmacology, Animal

Physiology and Physiological Chemistry,

Faculty of Veterinary Medicine,

Trakia University,

6000 Stara Zagora, Bulgaria,

phone: +35942699634,

e-mail: katvach@gbg.bg 